\title{
Nano-graphite as New Biosensing Material for Highly Sensitive and Selective Fluorescent Detection of Silver(I) Ion in Aqueous Solution
}

\section{Yin Wei*, JinlingYang, Guoqiang Shangguan, and Kai Zhang,}

Medical Examination Department, Jining Medical University, Jining, China

E-mail: weiyin1220@163.com

Keywords: Nano-graphite, Highly Sensitive, Selective Fluorescent Detection

\begin{abstract}
This paper describes a novel approach utilizing nano-graphite as an effective fluorescent sensing platform for highly sensitive and selective detection of $\mathrm{Ag}^{+}$in aqueous solution for the first time. Nano-graphite can effectively quench the fluorescence of dye-labeled cytosine-rich single-stranded DNA due to their strong $\pi-\pi$ stacking interactions; however, $\mathrm{Ag}^{+}$is able to specifically bind to the cytosine-cytosine (C-C) base pair in a DNA duplex, which does not adsorb on nano-graphite and thus retains the dye fluorescence. This sensing platform exhibits high selectivity and sensitivity toward $\mathrm{Ag}^{+}$versus other metal ions with a limit of detection of $500 \mathrm{pm}$.
\end{abstract}

\section{Introduction}

Silver ions are assigned to the highest toxicity class of heavy metal pollution. It is very important to monitor its content level in aquicolous ecosystems because a large amount of silver(I) ions is released to the environment annually from industrial wastes and emissions, especially to the sludge waste and even to surface waters [1-3]. A number of methodologies, such as electrothermal atomic absorption spectrometry (ETAAS) [4], voltammetry [5.6], inductively coupled plasma atomic emission spectrometry (ICP-AES) [7], inductively coupled plasma mass spectroscopy (ICP-MS) [8.9], potentiometry [10-12], and the fluorescence method have been reported in the past few years. However, these techniques suffer either from extensive, time consuming procedures or the use of sophisticated instrumentation.

On the other hand, the interactions between metal ions and nucleic acids have been paid considerable attention and have laid the foundation for oligonucleotide-based metal ions detection assay. It is well-known that $\mathrm{Ag}^{+}$can bind to two cytosine (C) residues of DNA to form the C- $\mathrm{Ag}^{+}-\mathrm{C}$ complex. The oligonucleotide (OND) is rich in cytosine and readily forms a hairpin structure in the $\mathrm{C}-\mathrm{Ag}^{+}-\mathrm{C}$ configuration in the presence of target $\mathrm{Ag}^{+}$ion. Various $\mathrm{Ag}^{+}$ion detection assays based on this property of $\mathrm{C}-\mathrm{Ag}^{+}-\mathrm{C}$ coordination chemistry have been developed in recent years.

In this paper, we demonstrate the first use of nano-graphite as a cheap, effective fluorescent sensing platform for $\mathrm{Ag}^{+}$detection, which is based on the noncovalent assembly of nano-graphite and dye-labeled cytosine -rich single-stranded DNA. This fluorescent sensing platform achieves a detection limit as low as $500 \mathrm{pM}$, which is much lower than that of carbon nanotubes and grapheneoxide system and exhibits excellent selectivity.

\section{Experiment}

The chemically synthesized oligonucleotide was purchased from Shanghai Sangon Biotechnology Co. Ltd. (Shanghai, China). Oligonucleotide sequence is listed as follows: 5'-ROX-CCT CCC TCC TTT TCC ACC CAC C-3'. DNA concentration was estimated by measuring the absorbance at $260 \mathrm{~nm}$. The nano-graphite $(10-15 \mathrm{~nm})$ was purchased from Nanjing XFNANO Materials Tech Co. Ltd. (Nanjing, China). It is important to mention that although nano-graphite tends to sink in the mixing solution of water and ethanol, they can be well-dispersed by shaking and kept stable during our measurements. All the other chemicals were purchased from Beijing Solarbio Co.Ltd. (Beijing, China) and used as received without further purification. The 
water used throughout all experiments was purified through a Millipore system. The total volume of each sample for fluorescence measur ement was $500 \mu \mathrm{L}$ in $10 \mathrm{mM}$ 3-(N-morpholino) propanesulfonic acid (MOPS) buffer containing $50 \mathrm{mM}$ NanNO3 (pH:7.5). The volume of nano-graphite used for each measurement was $40 \mu \mathrm{L}$, and the fluorescence quenching measurement was done after $20 \mathrm{~min}$ incubation in buffer. Transmission electron microscopy (TEM) measurements were made on a FEI Tecnai G2 F20 S-TWIN instrument (FEI, USA). Fluorescent emission spectra were recorded on a PerkinElmer LS55 Luminescence Spectrometer (PerkinElmer, UK).

\section{Results and Discussion}

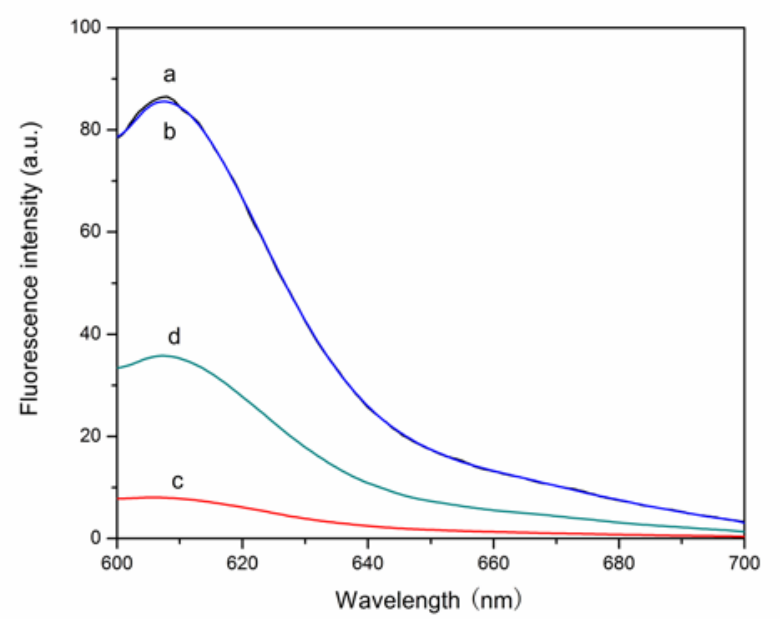

Figure 1. Fluorescence emission spectra of: (a) ssDNA; (b) ssDNA + $\mathrm{Ag}^{+}$; (c) ssDNA + nano-graphite; (d) ssDNA + nano-graphite $+\mathrm{Ag}^{+}$. Excitation was at $580 \mathrm{~nm}$ and the emission intensity was monitored at $606 \mathrm{~nm}$. All experiments were carried out in MOPS buffer (pH: 7.5) containing $50 \mathrm{mM}$ of $\mathrm{NanNO}_{3}$ and $100 \mathrm{nM}$ of ssDNA. ([ $\left.\mathrm{Ag}^{+}\right]$: $200 \mathrm{nM}$.)

Figure. 1 shows the fluorescence emission spectra of ROX-labeled ssDNA probe under different conditions. Upon the presence of the fluorescein-based dye, ssDNA shows strong fluorescence emission at $606 \mathrm{~nm}$ (curve a). The fluorescence of ssDNA was slightly influenced by $\mathrm{Ag}^{+}$(curve b). However, the presence of nano-graphite results in about $87 \%$ fluorescence quenching after 20 min incubation in MOPS solution (curve c), indicating that nano-graphite can efficiently quench the fluorescence of ROX, and the observed quenching phenomenon may be largely originated from the electron or energy transfer between the fluorophore and nano-graphite. In the presence of $200 \mathrm{nM}$ $\mathrm{Ag}^{+}$, the fluorescence intensity is about 4-fold higher than that without $\mathrm{Ag}^{+}$(curve d). The fluorescence enhancement is the result of the formation of dsDNA from ssDNA by $\mathrm{Ag}^{+}$, which detaches from nano-graphite and hampers the energy transfer between ROX and nano-graphite. We thus expect the fluorescence intensity changes of ssDNA/nano-graphite to provide a quantitative readout for $\mathrm{Ag}^{+}$.

To evaluate the sensitivity of this detection system, we collected emission spectra of ssDNA/nano-graphite in the presence of different concentrations of $\mathrm{Ag}^{+}$ranging from 0 to $400 \mathrm{nM}$, as shown in Fig 2. It is obvious that the fluorescence intensity of the mixture increases with the increase of $\mathrm{Ag}^{+}$concentration. The inset in Figure 2 shows the value of $\mathrm{F} / \mathrm{F} 0-1$ plotted against the concentration of $\mathrm{Ag}^{+}$, where $\mathrm{F}$ and $\mathrm{F} 0$ are the fluorescence intensity of ssDNA/nano-graphite with and without $\mathrm{Ag}^{+}$, respectively. Even at concentration as low as $5 \mathrm{nM}$, the fluorescence intensity is distinct from that without $\mathrm{Ag}^{+}$. The detection limit is estimated to be $500 \mathrm{pM}$ (three times the standard deviation of the blank solution), which is lower than the toxicity level of Ag+ in drinking water $(4.6 \times 10-7 \mathrm{M})$ defined by U.S. Environmental Protection Agency (EPA) [1]. The result indicates this fluorescent sensor holds a promising potential for practical applications. 


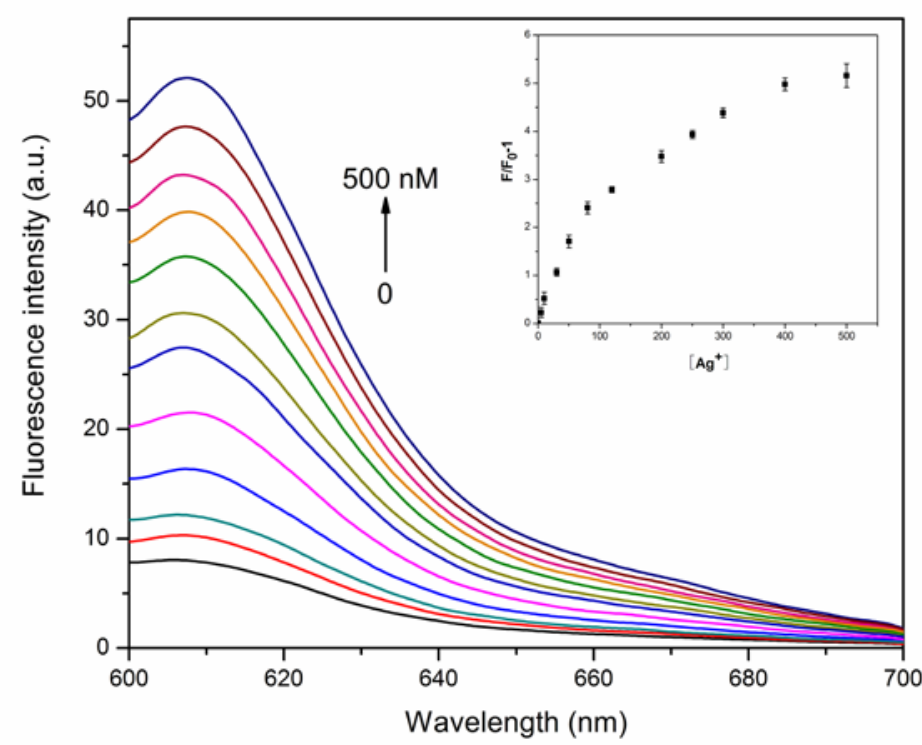

Figure 2. Fluorescence spectra of ssDNA/nano-graphite in the presence of different $\mathrm{Ag}^{+}$ concentrations (from bottom to top: 0, 5, 10, 30, 50, 80, 120, 200, 250, 300, 400, $500 \mathrm{nM}$ ). Inset: Inset: $\mathrm{F} / \mathrm{F} 0-1$ value plotted against the concentration of $\mathrm{Ag}^{+}$with error bar (standard deviation from the mean, $n=3$ ), where $F$ and F0 are the fluorescence intensity of ssDNA/nano-graphite with and without $\mathrm{Ag}^{+}$, respectively.

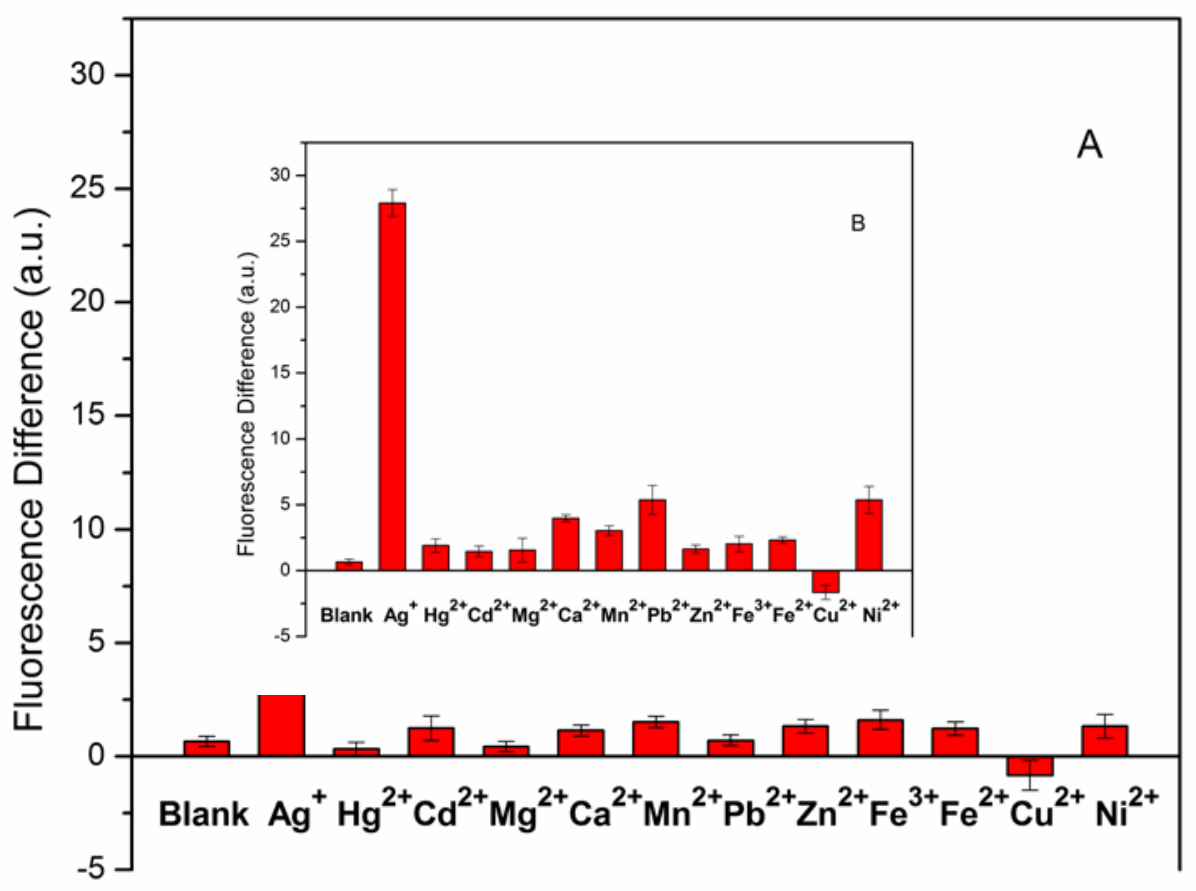

Figure 3. Fluorescence difference with error bars of ssDNA/nano-graphite between the blank and solutions containing different ions. Fluorescence difference $=$ FL Intensity (ssDNA/nano-graphite + Metal ions) - FL Intensity(ssDNA/ nano-graphite). (A: [other metal ions]: 2 uM; B: [other metal ions]: $20 \mathrm{uM}$ ). 
The selectivity of the present detection system for $\mathrm{Ag}^{+}$was also investigated. The enhanced fluorescence intensities were plotted against various environmentally relevant metal ions, including $\mathrm{Hg}^{2+}, \mathrm{Cd}^{2+}, \mathrm{Mg}^{2+}, \mathrm{Ca}^{2+}, \mathrm{Mn}^{2+}, \mathrm{Pb}^{2+}, \mathrm{Zn}^{2+}, \mathrm{Fe}^{3+}, \mathrm{Fe}^{2+}, \mathrm{Cu}^{2+}, \mathrm{Ni}^{2+}$ were examined. Fig 3 A shows the fluorescence difference between the blank and solutions containing $\mathrm{Ag}^{+}(200 \mathrm{nM})$ and other metal ions ( $2 \mathrm{uM})$. It can be seen that all the other metal ions presented negligible effects on the fluorescence of the ssDNA/ nano-graphite detection system. Furthermore, other metal ions at the concentration of $20 \mathrm{uM}$ were also investigated (Fig. $4 \mathrm{~B}$ ). Under such conditions, although the fluorescent sensing platform produces slightly bigger fluorescence differences in most cases than those observed at $2 \mathrm{uM}$, the use of $\mathrm{Ag}^{+}$still gives the best result. In all, these results clearly demonstrate that the ssDNA/nano-graphite system is highly selective toward $\mathrm{Ag}^{+}$over the other metal ions.

\section{Conclusions}

In conclusion, for the first time, we have demonstrated the successful use of nano-graphite as a cheap, effective fluorescent sensing platform for $\mathrm{Ag}^{+}$detection with high selectivity and sensitivity. This assay is based on the interaction between the target-induced conformational change of the ROX-ssDNA probe and nano-graphite quenching effects. We suggest that this assay offers several advantages. First, nano-graphite is cheaper and easier to produce than carbon nanotubes and grapheneoxide and nano-graphite can be easily synthesized with large quantities; Second, compared to SWCNT and GO sensing platforms, it exhibits a lower detection limit of $500 \mathrm{pM}$. We believe that this sensing platform will find great application in real environmental analysis.

\section{Acknowledgments}

The authors are grateful to the financial support from National Science Foundation of China: Investigation of Fast Pathogen Detection Based on Novel Macroporous Chips Technology (21275105), Jining Medical University Research Support Fund (JY2017KJ002), Jining Medical University Young Teachers Research Fund (JY2016KJ032Y), Jining City Science and Technology Development Project (2016-56-68).

\section{References}

[1] H. T. Ratte, Environ. Toxicol. Chem., 1999, 18, 89-108.

[2] K. Huang, W. Q Deng, R. Dai, X. Wang, Q. X. Q. Yuan, X. Jiang, X. Yuan and X. L. Xiong, Microchem. J., 2017, 135, 74-80.

[3] L. Chen, X. S. Zeng, H. F. Ju, X. W. He and Z. Z. Zhang, Microchem. J., 2000, 65, 129-135.

[4] J. L. Manzoori, H. Abdolmohammad-Zadeh and M. Amjadi, J. Hazard. Mater. , 2007, 144, 458-463.

[5] R. K. Shervedani and M. K. Babadi, Talanta, 2006, 69, 741-746.

[6] A. Mohadesi and M. A. Taher, Talanta, 2007, 71, 615-619.

[7] R. P. Singh and E. R. Pambid, Analyst, 1990, 115, 301-304.

[8] L. Yang and R. E. Sturgeon, J. Anal. At. Spectrom. , 2002, 17, 88-93.

[9] R. K. Katarina, T. Takayanagi, M. Oshima and S. Motomizu, Anal. Chim. Acta 2006, 558, 246-253.

[10] M. Shamsipur, M. Javanbakht, V. Lippolis, A. Garau, G. D. Filippo, M. R. Ganjali and A. Yari, Anal. Chim. Acta 2002, 462, 225-234.

[11] A. Ceresa, A. Radu, S. Peper, E. Bakker and E. Pretsch, Anal. Chem, 2002, 74, 4027-4036.

[12] K. Kimura, S. Yajima, K. Tatsumi, M. Yokoyama and M. Oue, Anal. Chem, 2000, 72, 5290-5294. 
